\title{
Advanced control solutions for enhanced resilience of modern power-electronic-interfaced distribution systems
}

\author{
Shiyuan WANG ${ }^{1}$, Payman DEHGHANIAN ${ }^{1}$ (D), Mohannad ALHAZMI', \\ Mostafa NAZEMI ${ }^{1}$
}

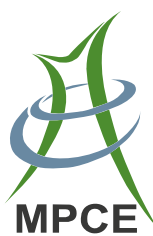

\begin{abstract}
Modern power delivery systems are rapidly evolving with high proliferation of power-electronic (PE)interfaced distributed energy resources (DERs). Compared to the conventional sources of generation, the PE-interfaced DERs, e.g., solar and wind resources, are attributed substantially different characteristics such as lower overload capability and limited frequency response patterns. This paper focuses on effective management and control mechanisms for PE-interfaced DERs in power distribution systems with high penetration of renewables, particularly under fault, voltage-sag, load variations, and other prevailing conditions in the grid. Aiming at the solutions to enhance the system performance resilience, we introduce an advanced model predictive control (MPC) based scheme to control the DER units, minimize the impact of transients and disruptions, speed up the response and recovery of particular metrics and parameters, and maintain an acceptable operation condition. The performance of the suggested control scheme is tested on a modified IEEE 34-bus test feeder, where the proposed solution
\end{abstract}

CrossCheck date: 18 April 2019

Received: 23 November 2018/Accepted: 18 April 2019/Published online: 22 July 2019

(C) The Author(s) 2019

Payman DEHGHANIAN

payman@gwu.edu

Shiyuan WANG

shiyuan1225@gwu.edu

Mohannad ALHAZMI

alhazmi@gwu.edu

Mostafa NAZEMI

mostafa_nazemi@gwu.edu

1 Department of Electrical and Computer Engineering, George Washington University, Washington 20052, USA demonstrates its effectiveness to minimize the system transient during faults, with an enhanced grid-edge and system-wide resilience characteristics in voltage profiles.

Keywords Distributed energy resource (DER), Model predictive control (MPC), Power electronics (PE), Protection, Resilience

\section{Introduction}

The elevated incidence and severity of high-impact lowprobability (HILP) events and outage-inducing weather and cyber patterns pose new challenges to the electricity grid and the increasingly electrified economy. The consequences can range from local disruptions in infrastructures resulting in or triggering cascading and widespread outages, network flow and voltage violations, etc. It has become more apparent that advanced planning, operation, and control solutions are needed ensuring resilience before, during and following such disturbances. Both long-term and short-term strategies for enhancing the grid resilience against extreme conditions have been addressed in [1]. In the former, enhancing the grid structural resilience is primarily the focus of concern and suggestions are made toward "grid hardening" plans through reinforcement, preventive maintenance of critical infrastructure, vegetation management, and efficient allocation of distributed energy resources (DERs) and other flexible energy resources, e.g., energy storage units [1-4]. In the latter, improving the operation resilience is targeted, through fast emergency response, remedial actions, defensive islanding scenarios and micro-grids control [5-8].

The rapid deployment of power-electronic (PE)-interfaced DERs in modern power distribution systems has 
introduced new opportunities and advanced solutions for power distribution system resilience against the HILP incidents as well as unpredictable faults and disturbances. Such resilience-driven solutions, however, have brought about new challenges and concerns which, in turn, call for the evolution of the traditional protection and control mechanisms that may no longer be optimal, if applicable at all. Depending on the depth and duration of the abnormalities (e.g., overvoltage conditions), the DER units might be disconnected from the grid, or keep being connected under permissible limits. Even if such conditions may last only for a short period of time, such events may potentially impose a risk of damage, performance degradation and lifetime reduction of PE-interfaced DERs and other grid connected devices. An effective protection and control scheme centered on PE devices at the edge connection of DERs to the distribution system is critical in order to prevent the disastrous consequences of the failures, ensuring resilience.

The effective utilization and control of advanced PE interfaces in DER-penetrated distribution systems and microgrids have been extensively investigated in [9-12]. The role of PE technologies on enhancing the microgrid resilience is discussed in [9]. Centered on PE converter technologies, a dynamic routing scheme is suggested in [10] to maximize the loadability in unbalanced distribution systems with hybrid AC and DC microgrids. Employing the voltage-shifting and slope-adjusting strategies, a distributed secondary control is introduced in [11] to eliminate the DC voltage deviation and improve the current sharing performance in DC microgrids. A control model for parallel operation of inverters is proposed in [13] to achieve the frequency and voltage control with no communication requirements between inverters. A control scheme for uninterruptible power supply (UPS) systems has been introduced in [14]. A circular chain control (3C) mechanism for inverters is introduced in [15] to achieve a highperformance current control. An extensive review on control schemes in multiple-cluster DC microgrids is explored in [12]. A cooperative distributed secondary/primary control paradigm for AC microgrids is introduced in [16]. Several other control strategies are proposed for highfidelity operation of parallel inverter interfaces $[13-15,17,18]$. Extensive fault analyses on the behaviors of inverter-interfaced DERs considering different control schemes are conducted and reported in [6], where the experiment results demonstrate that a current-controlled inverter typically has the ability to resist the fault current at a certain extent even in protection system failure scenarios. In most cases, protection devices can arrest the overvoltage surges and protective relays can isolate the electrical components from a long-term exposure to overvoltage conditions. However, protection failure does happen in practice. In particular, program logical control devices are widely utilized in current protective relays and circuit breakers to help the DER system undergo less severe conditions even during the faults or overvoltage scenarios. While the system can still continue operation under such prevailing conditions, the electrical power components will be stressed.

In this paper, an improved model predictive control (MPC) based mechanism is proposed, which primarily focuses on achieving the PE-interfaced DER performance resilience to voltage disruptions and overloading conditions during the faults. The entire effort is to create resilience culture on the PE-interfaced DER operation control and ensure that the voltage source inverters (VSIs) are able to function properly (overload free) and resiliently during the faults. The proposed MPC mechanism is featuring the following advantages:

1) Ensuring a fast dynamic response during both normal and abnormal conditions.

2) This MPC-based control scheme is embedded with two different strategies, achieving the flexibility in switching between balanced and unbalanced output modes accordingly.

3) Extending the application of MPC from supplying power to improving voltage resilience by mitigating the voltage degradation and regulation during abnormal conditions.

This paper is structured as follows: background information on power grid resilience and the basic principles of MPC are presented in Section 2. Section 3 is devoted to the PE-interfaced DER models. An MPC-based control scheme for voltage source inverter is proposed for gridconnected DER operation under normal and prevailing conditions in Section 4. To verify the promising performance of the proposed control scheme, numerical case studies are discussed in Section 5. And finally come the concluding remarks in Section 6.

\section{Background}

\subsection{Power grid resilience}

New North American Electricity Reliability Corporation (NERC) power system planning performance standard TPL-0014/0040a enforced in 2016 states that "studies shall be performed to assess the impact of the extreme events" [19]. The power grid is constantly exposed to potential hazards ranging from weather-driven natural disasters to malicious cybersecurity attacks. Due to numerous factors such as rapid deployment of intermittent renewable generation, growing demand to ensure higher quality 
electricity to end customers, and intensified public focus and regulatory oversights, safeguarding the national electric power grid and ensuring a continuous, reliable, and affordable supply of energy are among the top priorities for the electric power industry. Hence, resilience of the electricity grid and its capacity to withstand unexpected extreme events has become more and more critical for human well-being and every aspect of our economy [20-22].

Although well-known traditional reliability principles have been widely adopted in practice to make the grid operate securely and reliably under normal conditions and safely withstand credible contingencies ( $N-1$ criterion), the concept of "resilience" to HILP incidents has remained less clarified and unfocused. Higher power/energy capacity adequacy during extreme events, higher capacity accessibility (i.e. the extent of power and energy the grid can utilize from the generation sources), and higher reachability to such sources will render higher grid resilience during extreme events [21]. The concept of "resilience" has been quite explored in the literature in recent years. The word "resilience" is derived from the Latin word "resilire" highlighting the ability to rebound [23] and can be defined from many different perspectives. For instance, the National Infrastructure Advisory Council (NIAC) proposed a universal definition of infrastructure resilience in 2010: "the ability to reduce the magnitude and/or duration of disruptive events. The effectiveness of a resilient infrastructure depends on its ability to anticipate, absorb, adopt to and/or rapidly recover from a potentially disruptive event" [24]. Moreover, [25] defines the concept of resilience as the system ability to withstand the main interruption within an acceptable degradation performance and to recover within an acceptable time duration and composite risks and costs. The definition of "the system ability to prepare for and adapt to changing conditions, with robust performance and swift recovery" is proposed in [26]. Resilience is interpreted as the ability to prepare and plan for, absorb, recover from, and more successfully adopt to adverse events in [27]. Many other definitions of resilience can be found in economic systems [28], organizational systems [29], social systems [30] and complex networks [31, 32].

All the above definitions for resilience share a common goal of moderating the consequences of a severe shock to a system or infrastructure. Hence, monitoring the performance of a system subject to a disruption can help understand its resilience before, during, and after the event. A typical system performance evaluation framework following a disturbance is illustrated in Fig. 1, where the vertical axis represents the system performance (or a parameter of interest) over a period of time captured in the horizontal axis. The resilience evaluation starts at $t_{0}$, a

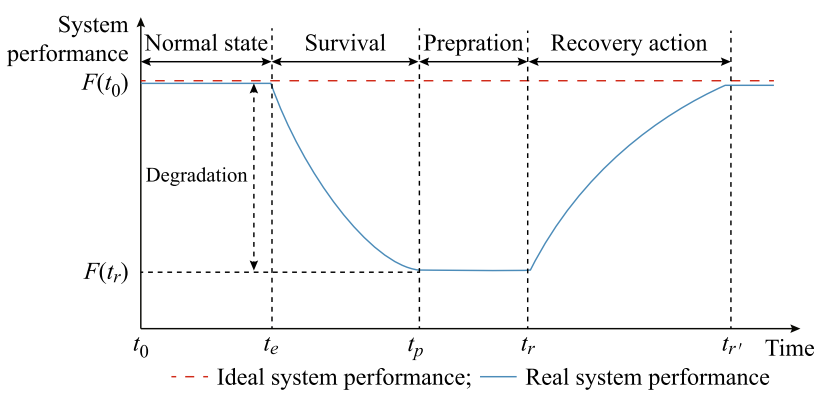

Fig. 1 General representation of a resilience evaluation framework over time

disturbance occurs at $t_{e}$, the performance index is degraded at $t_{e}$ and reaches to a minimum at $t_{p}$. Subsequently, the preparation and recovery process begins at $t_{p}$ and $t_{r}$, respectively, following which, the system performance elevates until it reaches its full functionality. As it can be seen, the temporal framework for resilience evaluation could be classified into four phases: normal state, survival, preparation, and recovery.

\subsection{MPC}

Over the past three decades, MPC analytics have been advanced and applied in different engineering disciplines such as process control, industrial control, as well as petrochemical processes, among many others [33-35]. With the relatively easy implementation and proven applicability to various multivariate systems, MPC offers a unique advantage to be applied for control of PE-interfaced DER infrastructure [36, 37].

Principally, MPC is a multi-variable control algorithm that uses an internal dynamic model of the process with a history of past control records and an optimization cost function $J$ over the receding prediction horizon under certain constrains. The MPC mathematical formulation can be generally expressed as follows:

1) Cost function

$\min _{u, y} \sum_{k=0}^{N-1} J\left(y_{k}(t+1), y_{k}(t), u_{k}(t), \eta_{k}(t)\right)$

2) System dynamics

$f\left(y_{k}(t+1), y_{k}(t), u_{k}(t), \eta_{k}(t)\right)=0$

3) Flow constraints

$g\left(y_{k}(t+1), y_{k}(t), u_{k}(t), \eta_{k}(t)\right) \leq 0$

4) Initial condition

$y_{k}=y_{k}(0)$

where $y_{k}$ is the predicted state of the system; $u_{k}$ is the input 


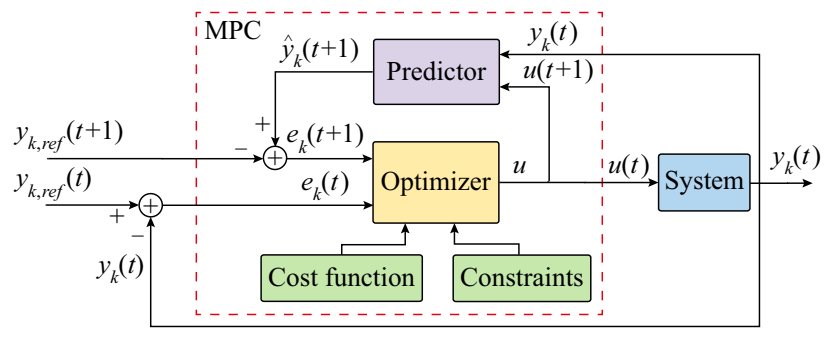

Fig. 2 General architecture of the MPC algorithm

with the set of its optimal values; $\eta_{\mathrm{k}}$ is the disturbance appearing in the system. The general architecture of the MPC process is demonstrated in Fig. 2, where $y_{k, r e f}$ is the reference state; $e_{k}$ is the error; $\hat{y}_{k}$ is the estimated state; $t$ is the current time; and $t+1$ is the future time step.

In the last decade, improvements on MPC have been majorly focused on speeding up its process to make it computationally friendly to applications with very fast sampling rate requirements [33]. Focusing on power grids with integration of PE-interfaced DER, MPC is a promising alternative to other classic power flow control mechanisms with pulse width modulation (PWM). Continuous control set-MPC (CCS-MPC) and finite control set-MPC (FCS-MPC) have been already implemented in PE-centered researches and developments [36, 38-40]. In the former, the continuous output of the predictive controller is used as a starting point to generate the switching states using the modulator, while in the latter, a limited number of switching states of converters are utilized with no dependence in the modulation stages. A discrete model is developed to predict the system behavior for every admissible actuation sequence up to the prediction horizon. The switching action that minimizes a predefined cost function is finally selected to be applied in the next sampling interval. Very recent MPC applications in PE-interfaced system controls are in the domains, including but not limited to distributed generation systems, active filters and power conditioning, drives, and UPS systems which are extensively reviewed in [35]. Different from the past researches on the application of MPC schemes, our proposed MPCbased control strategy extends the traditional MPCs from supplying power to improving voltage resilience during abnormal conditions. The proposed MPC ensures additional flexibility and a fast dynamic response during both normal and abnormal conditions. This performance is highlighted by the comparisons over the traditional schemes later in this paper.

\section{Modeling of PE-interfaced DERs}

\subsection{Waveform representation and modeling}

To simplify the analysis of a three-phase system and relax any dependence to a rotating reference frame commonly applied in the Park transformation [41], the Clarke transformation [42] is here employed first to convert a three-phase signal from the ABC-reference frame $\left(x_{A}(t)\right.$, $\left.x_{B}(t), x_{C}(t)\right)$ to components in a stationary $\alpha \beta$-frame, as follows:

$$
\begin{gathered}
x_{\alpha \beta}(t)=x_{\alpha}(t)+\mathrm{j} x_{\beta}(t) \\
\left\{\begin{array}{l}
{\left[\begin{array}{l}
x_{\alpha}(t) \\
x_{\beta}(t) \\
x_{\gamma}(t)
\end{array}\right]=C\left[\begin{array}{l}
x_{\mathrm{A}}(t) \\
x_{\mathrm{B}}(t) \\
x_{\mathrm{C}}(t)
\end{array}\right]} \\
\boldsymbol{C}=\left[\begin{array}{ccc}
\frac{2}{3} & -\frac{1}{3} & -\frac{1}{3} \\
0 & \frac{\sqrt{3}}{3} & -\frac{\sqrt{3}}{3} \\
\frac{1}{3} & \frac{1}{3} & \frac{1}{3}
\end{array}\right]
\end{array}\right.
\end{gathered}
$$

Clarke transformation will result in a rotating phasor. During unbalanced conditions, both the magnitude and angular speed of the phasor experience periodical changes $[43,44]$ that can be used to represent the grid operation conditions.

\subsection{VSI switching models}

In general, the single-phase model of a PE-interfaced DER system can be represented by a DC voltage source, a one-leg inverter (with two switching gates), and an LC filter. The blue arrow in Fig. 3 represents the measured data/control signal flow (discrete data format). A traditional one-phase VSI model embedded with an MPC controller with voltage, current, or power control strategies is illustrated in Fig. 3, where $R_{f}, L_{f}, C_{f}$ are the resistance, inductance and capacitance of AC filter, respectively. $S_{p h}$ stands for the switching signal; $V_{\mathrm{dc}}$ is the DC link voltage; $v_{o}$ and $i_{o}$ are the output voltage and current of AC filter, respectively; $i_{f}$ is the inductor current; $i_{c}$ is the capacitor current; $v_{i}$ is the VSI output voltage; and $v_{c}$ and $i_{c}$ are the voltage and current across the capacitor of AC filter. According to Fig. 4, the switch signal $S_{p h}$ for each phase is fed into the gates and the three-phase output voltage can be described as follows:

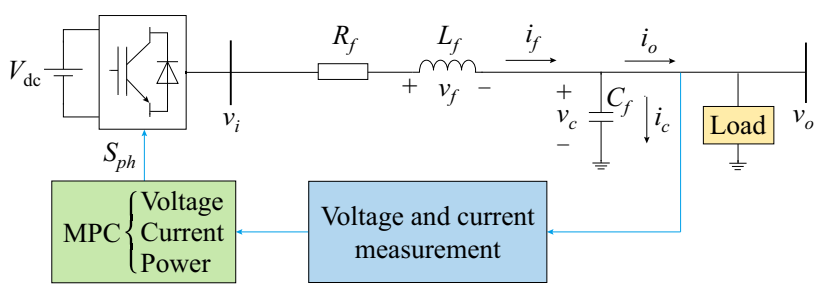

Fig. 3 Single-line model of the PE-interfaced DER system with MPC controller 
$V_{n}= \begin{cases}\frac{2}{3} V_{\mathrm{dc}} \mathrm{e}^{\mathrm{j}(n-1) \frac{\pi}{3}} & n=1,2, \ldots, 6 \\ 0 & n=0,7\end{cases}$

The switching combination $\left[S_{\mathrm{A}}, S_{\mathrm{B}}, S_{\mathrm{C}}\right]$ for the above eight possible output voltages are $[000,110,110,010,011$, $001,101,111]$, with " 1 " representing the ON status for the gate switches and " 0 " otherwise. The output voltage and current of the DER system can be mathematically expressed as:

$v_{i}=v_{o}+R_{f} i_{f}+L_{f} \frac{\mathrm{d} i_{f}}{\mathrm{~d} t}$

$i_{o}=i_{f}-i_{c}$

In a grid-connected operation, i.e., $v_{o}=v_{g}$, where $v_{g}$ is the voltage at the grid-connected node, the states corresponding to the two switching gates are unalikeone is ON while the other is OFF. To ensure an acceptable power quality, a suitable set of capacitors are selected and deployed during normal grid-connected operation conditions. This will yield a rise in the voltage, and therefore, an inductance load is added to compensate the reactive power provided by the capacitor.

\subsection{Three-dimensional space vector modulation (3D SVM)}

In order to capture the neutral current resulting from a nonlinear load and/or unbalanced sources in the grid, a four-leg inverter model is suggested and a 3D SVM scheme is proposed in [45]. The four-leg inverter model can be achieved by including another pair of switching gates into a three-leg inverter model previously described in Fig. 4. The neutral branch is characterized via an inductor $L_{n}$ to reduce the switch ripples; it is connected to the common point of a three-phase LC filter to smoothen the unbalanced output. The configuration of the four-leg inverter with an LC filter employed in this paper is demonstrated in Fig. 5 and the corresponding switching patterns are listed in Table 1.

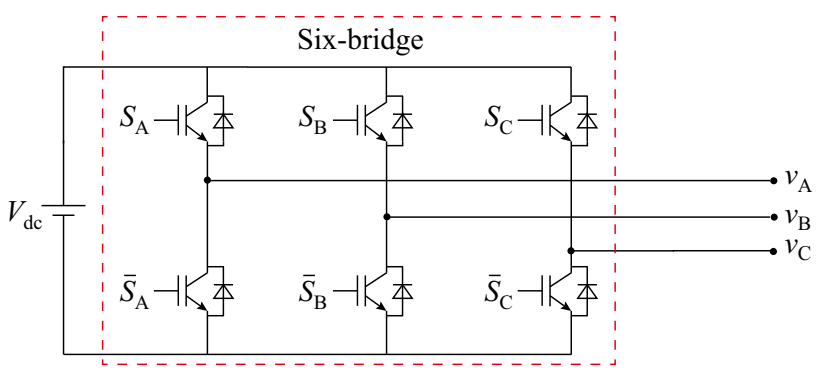

Fig. 4 Diagram of a three-leg six-bridge VSI

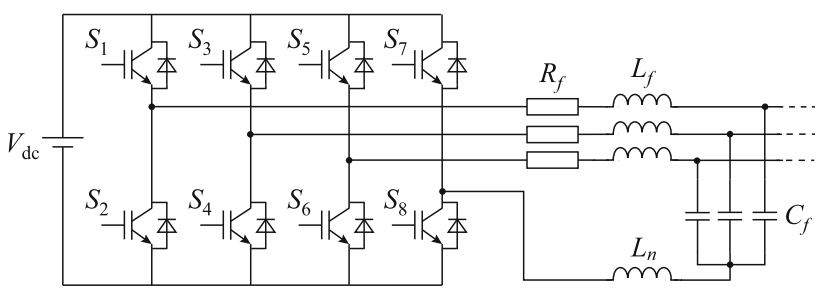

Fig. 5 Diagram of a four-leg inverter with an LC filter

Table 1 Switching combinations and the associated inverter output voltages in the $\alpha \beta \gamma$-coordinates

\begin{tabular}{lllllllll}
\hline$n$ & 0 & 1 & 2 & 3 & 4 & 5 & 6 & 7 \\
\hline $\mathrm{S}$ & 0000 & 0001 & 0010 & 0011 & 0100 & 0101 & 0110 & 0111 \\
$V_{\alpha}$ & 0 & 0 & $\frac{2 V_{d c}}{3}$ & $\frac{V_{d c}}{3}$ & $\frac{-V_{d c}}{3}$ & $\frac{-V_{d c}}{3}$ & $\frac{V_{d c}}{\sqrt{3}}$ & $\frac{V_{d c}}{3}$ \\
$V_{\beta}$ & 0 & 0 & 0 & $\frac{V_{d c}}{\sqrt{3}}$ & $\frac{V_{d c}}{\sqrt{3}}$ & 0 & $\frac{-V_{d c}}{\sqrt{3}}$ & $\frac{-V_{d c}}{\sqrt{3}}$ \\
$V_{\gamma}$ & 0 & $-V_{d c}$ & $\frac{-V_{d c}}{3}$ & $\frac{-2 V_{d c}}{3}$ & $\frac{-2 V_{d c}}{3}$ & $\frac{-V_{d c}}{3}$ & $\frac{-2 V_{d c}}{3}$ & $\frac{-V_{d c}}{3}$ \\
\hline$n$ & 8 & 9 & 10 & 11 & 12 & 13 & 14 & 15 \\
\hline $\mathrm{S}$ & 1000 & 1001 & 1010 & 1011 & 1100 & 1101 & 1110 & 1111 \\
$V_{\alpha}$ & 0 & 0 & $\frac{2 V_{d c}}{3}$ & $\frac{V_{d c}}{3}$ & $\frac{-V_{d c}}{3}$ & $\frac{-V_{d c}}{3}$ & $\frac{V_{d c}}{\sqrt{3}}$ & $\frac{V_{d c}}{3}$ \\
$V_{\beta}$ & 0 & 0 & 0 & $\frac{V_{d c}}{\sqrt{3}}$ & $\frac{V_{d c}}{\sqrt{3}}$ & 0 & $\frac{-V_{d c}}{\sqrt{3}}$ & $\frac{-V_{d c}}{\sqrt{3}}$ \\
$V_{\gamma}$ & $V_{d c}$ & 0 & $\frac{V_{d c}}{3}$ & $\frac{2 V_{d c}}{3}$ & $\frac{V_{d c}}{3}$ & $\frac{2 V_{d c}}{3}$ & $\frac{V_{d c}}{3}$ & $\frac{2 V_{d c}}{3}$
\end{tabular}

$S:$ On/Off (1/0) status in a binary format

$n$ : Decimal value of $S$ for indexing purpose

The switching combinations can be represented by an ordered set of $S=\left[S_{1}, S_{3}, S_{5}, S_{7}\right]$, where the status of the lower-level gates is always opposite to that of the upperlevel gates to avoid the short-circuit scenarios. It should be noted that $V_{\gamma}$ in Table 1 represents the zero-sequence component and corresponds to the neutral current. Therefore, the voltage control vector trajectory in the case of a balanced load is a circle in the $\alpha \beta$-frame, while it rotates into a $3 \mathrm{D}$ space in the case of an unbalanced load. The transformation of the 2D SVM to the 3D SVM in the $\alpha \beta$ frame is graphically illustrated in Fig. 6.

\section{Grid-connected operation and control of PE- interfaced DERs}

The proposed control scheme is centered on the active power and reactive power control, which is mainly applied in grid-connected operations. Dealing with the abnormal operation conditions is a challenge in islanded operation modes. During islanded operation modes, the proposed control scheme can still be applied, but together with other energy resources, where the proposed scheme will operate 


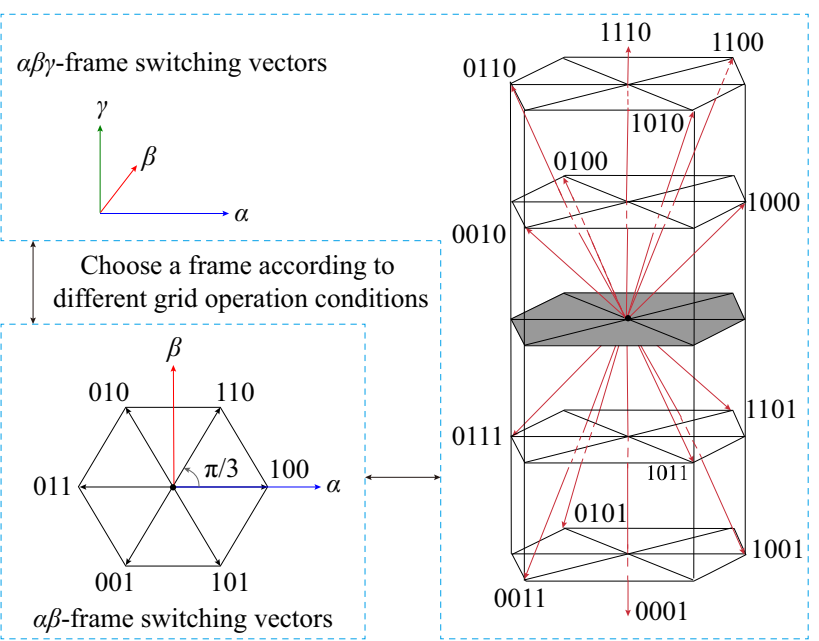

Fig. 6 Switching patterns and voltage control vectors of a four-leg inverter in $\alpha \beta \gamma$-frame

as an auxiliary power source (not the main power supply) providing voltage and frequency regulation benefits. The control scheme in this section focuses on grid-connected operation modes in two scenarios in normal and abnormal conditions, respectively.

\subsection{DER control in normal operation and balanced load change scenarios}

In normal operation scenarios where the PE-interfaced DER is grid-connected and there is no fault in the system, there still exists a possibility of balanced load changes. The proposed control scheme is designed to react to such scenarios and inject power to the grid accordingly, ensuring the generation-load balance as well as a resilient local system-wide voltage regulation. The DER instantaneous output active and reactive powers in balanced distribution systems are as follows:

$S=P+\mathrm{j} Q=\boldsymbol{V}_{g, \mathrm{ABC}}^{\mathrm{T}} \boldsymbol{I}_{f, \mathrm{ABC}}^{*}$

$\left\{\begin{array}{l}\boldsymbol{V}_{g, \mathrm{ABC}}=\left[\begin{array}{lll}V_{g, \mathrm{~A}} & V_{g, \mathrm{~B}} & V_{g, \mathrm{C}}\end{array}\right]^{\mathrm{T}} \\ \boldsymbol{I}_{f, \mathrm{ABC}}=\left[\begin{array}{lll}I_{f, \mathrm{~A}} & I_{f, \mathrm{~B}} & I_{f, \mathrm{C}}\end{array}\right]^{\mathrm{T}}\end{array}\right.$

$P=\operatorname{Re}\left\{\boldsymbol{V}_{g, \mathrm{ABC}}^{\mathrm{T}} \boldsymbol{I}_{f, \mathrm{ABC}}^{*}\right\}=\frac{3}{2}\left(v_{g, \alpha} i_{f, \alpha}+v_{g, \beta} i_{f, \beta}\right)$

$Q=\operatorname{Im}\left\{\boldsymbol{V}_{g, \mathrm{ABC}}^{\mathrm{T}} \boldsymbol{I}_{f, \mathrm{ABC}}^{*}\right\}=\frac{3}{2}\left(v_{g, \beta} i_{f, \alpha}-v_{g, \alpha} i_{f, \beta}\right)$

where $\boldsymbol{V}_{g, \mathrm{ABC}}$ and $\boldsymbol{I}_{f, \mathrm{ABC}}$ are the three-phase grid connected node voltage phasor vector and three-phase VSI output current phasor vector, respectively; $*$ donates the complex conjugate and $\mathrm{T}$ donates the transposition operation. To control the power injection, the state space of the LC filter is needed which can be developed through derivatives of the DER power outputs. According to the equation set (12), (13), the derivatives are obtained as follows:

$$
\begin{aligned}
& \frac{\mathrm{d} P}{\mathrm{~d} t}=\frac{3}{2}\left(\frac{\mathrm{d} v_{g, \alpha}}{\mathrm{d} t} i_{f, \alpha}+v_{g, \alpha} \frac{\mathrm{d} i_{f, \alpha}}{\mathrm{d} t}+\frac{\mathrm{d} v_{g, \beta}}{\mathrm{d} t} i_{f, \beta}+v_{g, \beta} \frac{\mathrm{d} i_{f, \beta}}{\mathrm{d} t}\right) \\
& \frac{\mathrm{d} Q}{\mathrm{~d} t}=\frac{3}{2}\left(\frac{\mathrm{d} v_{g, \beta}}{\mathrm{d} t} i_{f, \alpha}+v_{g, \beta} \frac{\mathrm{d} i_{f, \alpha}}{\mathrm{d} t}-\frac{\mathrm{d} v_{g, \alpha}}{\mathrm{d} t} i_{f, \beta}-v_{g, \beta} \frac{\mathrm{d} i_{f, \alpha}}{\mathrm{d} t}\right)
\end{aligned}
$$

Assuming the initial phase to be zero in a balanced distribution system, the output voltage in the $\alpha \beta$-frame (5) and the derivatives can be expressed as follows:

$\vec{v}_{\alpha \beta}=|v| \mathrm{e}^{\mathrm{j} \omega \mathrm{t}}=|v| \cos \omega t+\mathrm{j}|v| \sin \omega t$

$\frac{\mathrm{d} v_{g, \alpha}}{\mathrm{d} t}=\frac{\mathrm{d}\left(\left|v_{g, \alpha \beta}\right| \cos \omega t\right)}{\mathrm{d} t}=-\omega v_{g, \beta}$

$\frac{\mathrm{d} v_{g, \beta}}{\mathrm{d} t}=\frac{\mathrm{d}\left(\left|v_{g, \alpha \beta}\right| \sin \omega t\right)}{\mathrm{d} t}=\omega v_{g, \alpha}$

Employing (8), (17), and (18) to substitute all the derivatives in (14) and (15), we can obtain:

$\frac{\mathrm{d} \boldsymbol{x}}{\mathrm{d} t}=\boldsymbol{A} \boldsymbol{x}+\frac{3}{2 L_{f}} \boldsymbol{B} \boldsymbol{u}+\boldsymbol{D}$

$\left\{\begin{array}{l}\boldsymbol{x}=\left[\begin{array}{l}P \\ Q\end{array}\right] \\ \boldsymbol{u}=\left[\begin{array}{l}v_{f, \alpha} \\ v_{f, \beta}\end{array}\right] \\ \boldsymbol{A}=\left[\begin{array}{cc}-\frac{R_{f}}{L_{f}} & -\omega \\ \omega & -\frac{R_{f}}{L_{f}}\end{array}\right] \\ \boldsymbol{B}=\left[\begin{array}{cc}v_{g, \alpha} & v_{g, \beta} \\ v_{g, \beta} & -v_{g, \alpha}\end{array}\right] \\ \boldsymbol{D}=\left[\begin{array}{c}\left|v_{g, \alpha \beta}\right|^{2} \\ 0\end{array}\right]\end{array}\right.$

The discrete-time control model of the PE-interfaced DER system can be expressed as:

$$
\begin{aligned}
& \boldsymbol{x}[k+1]=\boldsymbol{A}_{d} \boldsymbol{x}[k]+\frac{3}{2 L_{f}} \boldsymbol{B}_{d}[k] \boldsymbol{u}[k]+\boldsymbol{D}[k] \\
& \left\{\begin{array}{l}
\boldsymbol{A}_{d}=\mathrm{e}^{A T_{s}} \\
\boldsymbol{B}_{d}[k]=\int_{0}^{T_{s}} \mathrm{e}^{\boldsymbol{A} \tau} \boldsymbol{B}[k] \mathrm{d} \tau
\end{array}\right.
\end{aligned}
$$

where $k$ is the discrete time variable; and $T_{s}$ is the sampling interval. If $T_{s}$ is sufficiently small, (22) can be approximated by the following series.

$$
\mathrm{e}^{A T_{s}}=I+A T_{s}+\frac{\left(A T_{s}\right)^{2}}{2 !}+\cdots+\frac{\left(A T_{s}\right)^{n}}{n !} \approx I+A T_{s}
$$


where $\boldsymbol{I}$ is the identity matrix. This approximation can significantly reduce the computational burden and programming complexity of (21) and (22). Assuming that the grid voltage is constant during a very short sampling interval, the future value of the active and reactive power can be predicted by (21). The next step will be to evaluate the effect of each voltage vector on $v_{i}$ in (7) and select the one which results in a minimum value of the following cost function.

$$
J=\left(P_{\text {ref }}-P[k+1]\right)^{2}+\left(Q_{r e f}-Q[k+1]\right)^{2}
$$

where $P$ is the real power; $P_{r e f}$ is the reference real power; $Q$ is the reactive power; Qref is the reference reactive power. The selected voltage vector will be the VSI gate switching pattern in the next switching interval. The block diagram of the proposed control scheme under a normal grid-connected operation of PE-interfaced DERs is demonstrated in Fig. 7.

\subsection{DER control in abnormal conditions and voltage disturbances}

In abnormal operation scenarios where either there is a fault and voltage sag or a sudden variation of the unbalanced load happens, the voltage at the grid-edge connection of the PE-interfaced DER will experience distortions. The RMS voltage at each phase is then crucial to spatiotemporally detect the faults or large load variations. In an unbalanced load scenario, the VSI needs to adjust the DER output power to regulate the voltage accordingly. The VSI has the ability to restrict the DER power injection to the grid to handle the overvoltage conditions, or increase the DER output power to mitigate the voltage dips. In such unbalanced load scenarios, the VSI's forth leg is active and the circuit model is illustrated in Fig. 5, which is mathematically expressed as:

$\boldsymbol{v}_{i, \mathrm{ABC}}=L_{f} \frac{\mathrm{d} i_{f, \mathrm{ABC}}}{\mathrm{d} t}+R_{f} \boldsymbol{i}_{f, \mathrm{ABC}}+\boldsymbol{v}_{g, \mathrm{ABC}}-L_{n} \frac{\mathrm{d} i_{n}}{\mathrm{~d} t}$ $i_{n}=-\sum \boldsymbol{i}_{f, \mathrm{ABC}}-C_{f} \frac{\mathrm{d}\left(\sum \boldsymbol{v}_{g, \mathrm{ABC}}\right)}{\mathrm{d} t}$

where $\boldsymbol{v}_{i, \mathrm{ABC}}$ and $\boldsymbol{i}_{f, \mathrm{ABC}}$ stand for the three-phase inverter output voltages and currents, respectively; $\boldsymbol{v}_{g, \mathrm{ABC}}$ and $\boldsymbol{i}_{o, \mathrm{ABC}}$ are the three-phase voltages at the grid-connected node and the output current injected to the grid; $i_{n}$ and $L_{n}$ are the neutral current and the inductor. The transformation matrix $\boldsymbol{C}$ in (6) is applied and use (9) to simplify the voltage derivative in (26). Then, the $\alpha \beta \gamma$-frame system function is expressed as:

$$
\begin{aligned}
& \frac{\mathrm{d}_{f, \alpha \beta \gamma}}{\mathrm{d} t}=\boldsymbol{A}_{p} \boldsymbol{i}_{f, \alpha \beta \gamma}+\boldsymbol{B}_{p}\left[\boldsymbol{v}_{i, \alpha \beta \gamma}-\boldsymbol{v}_{g, \alpha \beta \gamma}\right]+\boldsymbol{D}_{p} \frac{\mathrm{d} \boldsymbol{i}_{o, \alpha \beta \gamma}}{\mathrm{d} t} \\
& \left\{\begin{array}{l}
\boldsymbol{A}_{p}=-\left[\frac{L_{f}}{R_{f}} \boldsymbol{I}+\frac{2 L_{n}}{R_{f}} \boldsymbol{C} \boldsymbol{G} \boldsymbol{C}^{-1}\right]^{-1} \\
\boldsymbol{B}_{p}=-\frac{\boldsymbol{A}_{p}}{R_{f}} \\
\boldsymbol{D}_{p}=\left[\frac{L_{f}}{L_{n}} \boldsymbol{C}^{-1}+2 \boldsymbol{G} \boldsymbol{C}^{-1}\right]^{-1} \boldsymbol{G} \boldsymbol{C}^{-1}
\end{array}\right.
\end{aligned}
$$

where the subscript $\alpha \beta \gamma$ denotes the $\alpha \beta \gamma$-frame for both voltage and current. $\boldsymbol{G}$ is an all-one matrix. Assuming the sampling rate is sufficiently large, one can use (29) to approximate $\mathrm{d} \boldsymbol{i}_{g, \alpha \beta \gamma} / \mathrm{d} t$ in discrete time for simplicity. Then applying (22) to (27), the discrete state-space system function is achieved as:

$$
\begin{aligned}
& \frac{\mathrm{d} y}{\mathrm{~d} t} \approx \frac{y[k+1]-y[k]}{T_{s}} \\
& i_{f, \alpha \beta \gamma}[k+1]=A_{p d} i_{f, \alpha \beta \gamma}[k]+B_{p d} v_{i, \alpha \beta \gamma}[k] \\
& \quad-B_{p d} v_{g, \alpha \beta \gamma}[k]+\frac{D_{p d}}{T_{s}}\left[i_{o, \alpha \beta \gamma}[k]-i_{o, \alpha \beta \gamma}[k-1]\right]
\end{aligned}
$$

where $A_{p d}, B_{p d}, D_{p d}$ are the discrete-time system models of $\boldsymbol{A}_{p}$, $\boldsymbol{B}_{p}, \boldsymbol{D}_{p}$, respectively. Assuming that the inverter output current is still within the desired thresholds under such abnormal conditions and the PE-interfaced DER is functioning in a gridconnected operation mode, the predicted nominal current $i_{f, \alpha \beta \gamma}[k+1]$ is obtained via (30), where $v_{g}[k]$ acts as the future value of $v_{g}[k+1]$ as a presumed future grid voltage. Finally, predicted power are calculated in $\alpha \beta \gamma$-frame as follows:

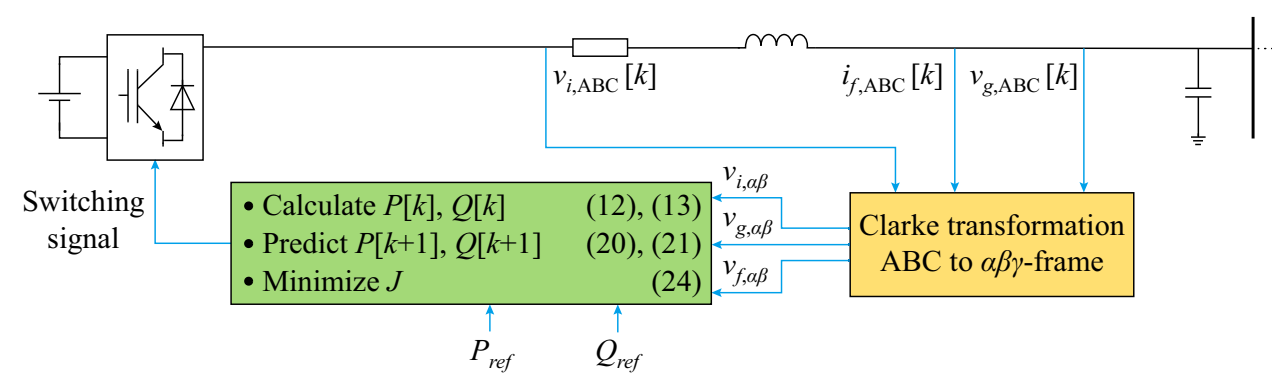

Fig. 7 Diagram of the proposed MPC-based DER control scheme during normal operation conditions 


$$
\left[\begin{array}{c}
P \\
Q \\
P_{0}
\end{array}\right]=\frac{3}{2}\left[\begin{array}{ccc}
v_{g, \alpha} & v_{g, \beta} & 0 \\
v_{g, \beta} & -v_{g, \alpha} & 0 \\
0 & 0 & v_{g, \gamma}
\end{array}\right]\left[\begin{array}{c}
i_{f, \alpha} \\
i_{f, \beta} \\
i_{f, \gamma}
\end{array}\right]
$$

Similar to the analytics introduced for DER control in normal operation conditions, the predicted output power under disturbances at time instant $k$ can be calculated using different voltage control vectors listed in Table 1. The MPC cost function (24) for DER control in faults and disturbance scenarios now becomes:

$J=\left(P_{r e f}-P\right)^{2}+\left(Q_{r e f}-Q\right)^{2}+\left(P_{0 r e f}-P_{0}\right)^{2}$

where $P_{0}$ is the zero sequence power; $P_{0 \text { ref }}$ is the reference zero sequence power. Here, the cost function appears to be a three-dimension function in the Cartesian coordinate system. Note that the $2 \mathrm{D}$ voltage switching patterns previously discussed for normal operating conditions are no longer sufficient to provide the input voltage vectors as desired. Suppose the DER unit is not able to regulate the grid connected node voltage to a desired value $\left|V_{g, r e f}\right|$ following a disturbance, e.g., a huge load loss in the distribution system triggered by a fault. Then, it is theoretically possible that the VSI may function as a converter, absorbing power from the grid. In such scenarios, if the DER cannot absorb the required power from the grid and the difference between the desired and actual power exceeds a preset threshold, the DC-link voltage needs to be regulated as well. To simplify the simulation process: (i) the DC link is modeled as an ideal DC voltage source; (ii) all the phasor amplitudes are captured from a low-pass filter since the measurements are also vulnerable during disturbances and transients; (iii) a finite set of DC-link voltage values are selected reflecting the converter functionality of the DER in such scenarios. A diagram of the proposed scheme for PE-interfaced DER control during abnormal operation conditions is demonstrated in Fig. 8. The overall architecture of the proposed scheme for DER control is presented in Fig. 9.

\section{Test cases and numerical analysis}

\subsection{Test platform, configuration and assumptions}

A modified IEEE 34-bus test feeder is employed as the test platform in this paper, the single-line diagram of which is illustrated in Fig. 10. The simulations are conducted in EMTDC/PSCAD. All tap chargers within the voltage regulating transformers are set to be fixed, and hence, the voltage across the grid will not be affected by other regulating devices in the grid (i.e., all other voltage regulators are set off, except the one proposed in this paper for the DERs). The DER is assumed to be located at Bus 852, and the data and measurements are captured from a PMU-installed location at Bus 850 serving as the global performance evaluation point. With micro-PMUs or other monitoring devices installed at different nodes across the network, the proposed control scheme does require a central master controller which monitors the grid, coordinates the switches as well as the DER controller directly, and ensures a balanced load across the network through communication channel. The LC filter is built of $R_{f}=0.005 \Omega$, $L_{f}=0.02 \mathrm{mH}, L_{n}=100 \mathrm{mH}$, and $C_{f}=960 \mu \mathrm{F}$. The maximum voltage of the DC-link is set to be $V_{\mathrm{dc}}=1 \mathrm{kV}$, and the DER rated power is $0.5 \mathrm{MVA}$.

\subsection{Test cases and scenarios}

\subsubsection{DER control under disturbances}

When there is a fault in the system (potentially due to a HILP event), the grid protective devices will detect the

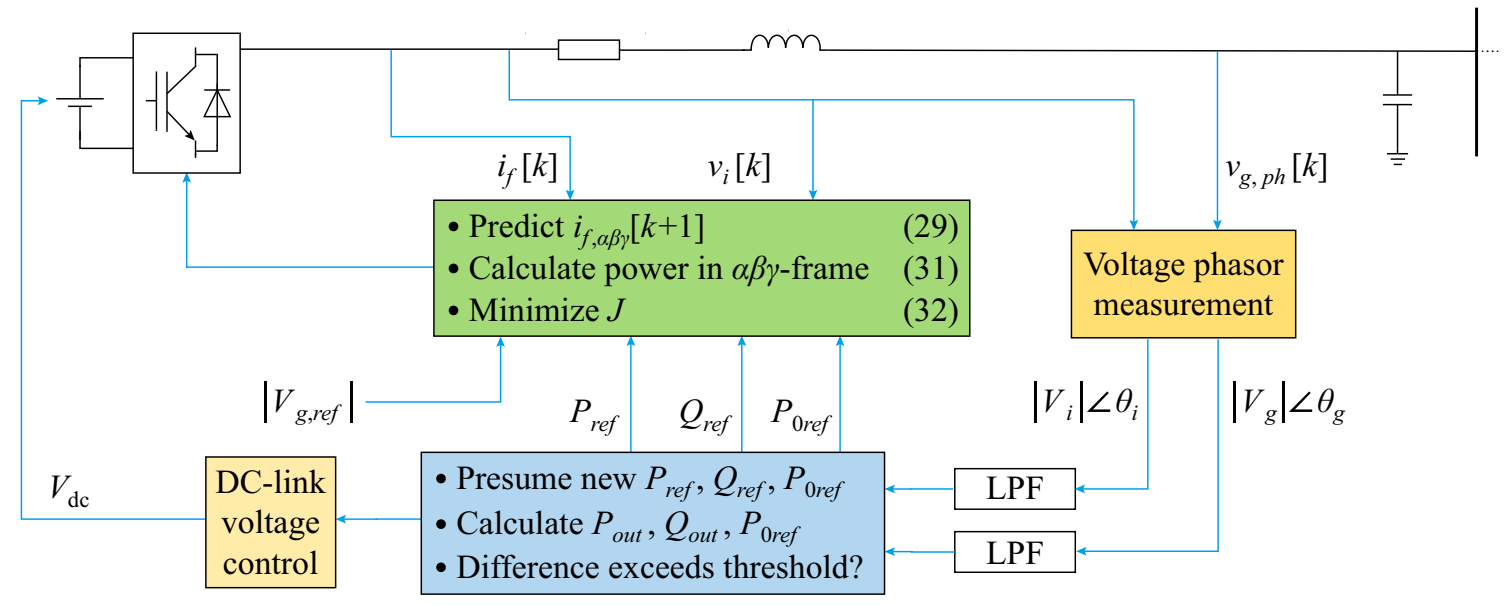

Fig. 8 Diagram of the proposed MPC-based DER control scheme during abnormal operation conditions 


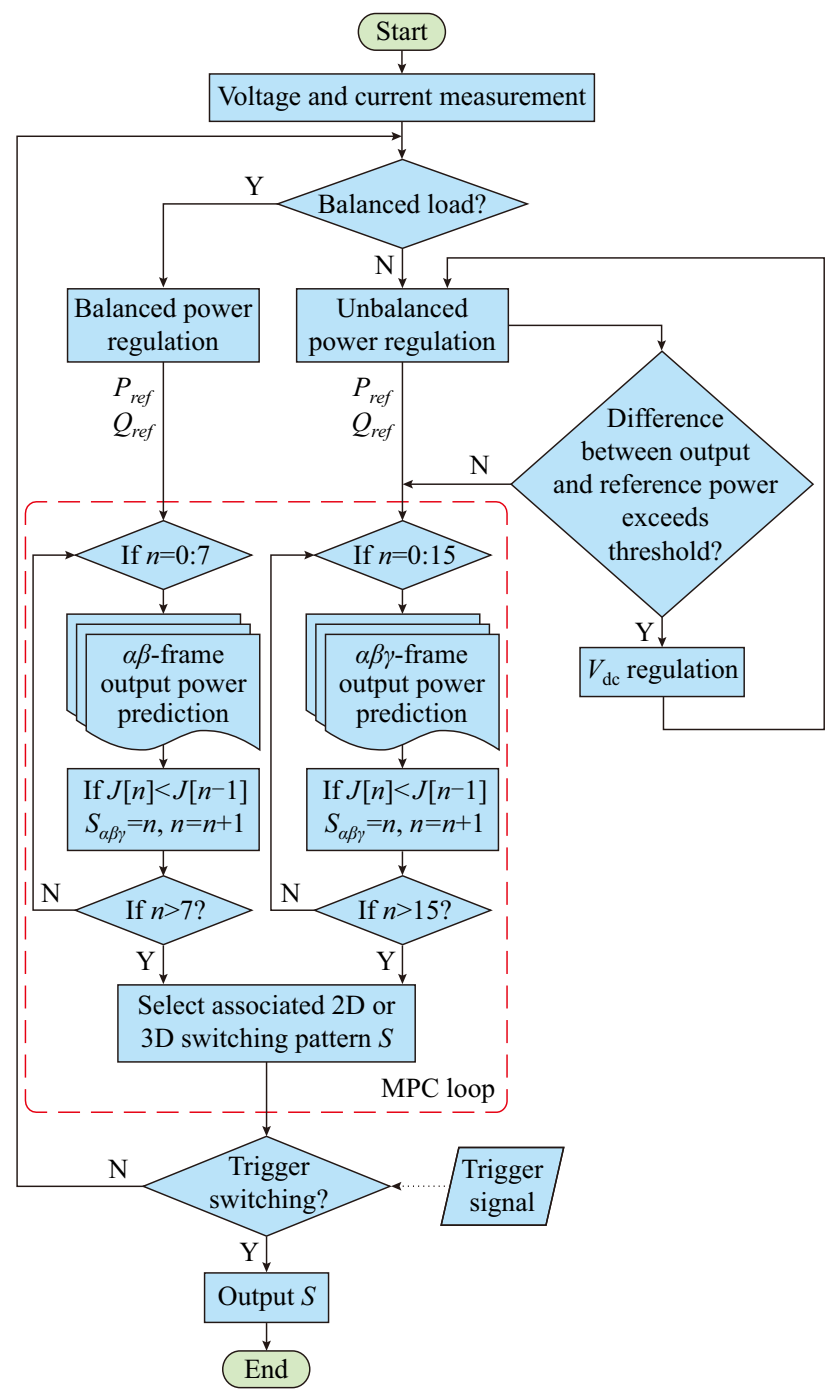

Fig. 9 Overall architecture of the proposed control scheme for PEinterfaced DERs

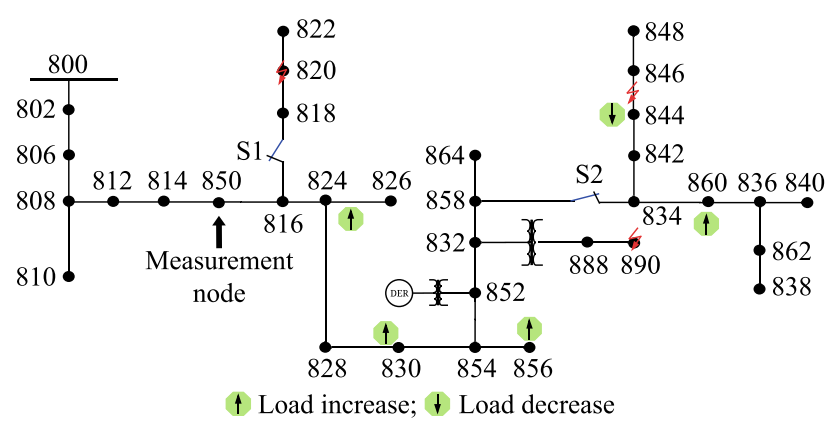

Fig. 10 Single-line configuration of the test system

fault and the normally-closed breakers will be signaled to switch ON. Consequently, a sudden load drop may be observed. As a result, a voltage rise may appear in the non-faulted sections of the grid. The voltage regulation needs to be enforced in such scenarios and the PE-interfaced DER needs to adapt to the voltage change by properly adjusting its output power.

In order to verify the effectiveness of the proposed control scheme for PE-interfaced DER operation in abnormal conditions and voltage disturbances, the following two test scenarios with corresponding test cases are studied and extensively analyzed:

- Test Scenario 1: voltage regulation at the edge busthrough which the DER is connected to the grid-, when the system experiences a fault and the DER is grid-connected. The protection devices are functional and able to swiftly clear the fault.

1) A single-line-to-ground (SLG) fault occurs in Phase A at Bus 820. The normally-closed switch 1 (pinpointed in Fig. 10) is opened in response and isolates the fault.

2) A three-phase-to-ground ( $3 \Phi \mathrm{G})$ fault occurs on the distribution line connecting Bus 834 to Bus 858 . The normally-closed switch 2 (pinpointed in Fig. 10) is opened and isolates the fault.

- Test Scenario 2: voltage regulation at the edge bus, and there is a high-resistance fault at the low-voltage section of the grid, where the breakers may fail to detect and clear the fault.

3) A 3-phase high-resistance fault occurs at Bus 890, and remains undetected by the protective devices in the grid.

\subsubsection{DER control in normal operation conditions}

This section verifies the ability of the proposed DER control scheme for voltage regulation during normal operation conditions. In such scenarios, load variations may occur, resulting in minor voltage changes across the grid. The voltage regulation and control may need to be activated so as to adjust the DER output power injection corresponding to load variation scenarios.

To verify the performance of the proposed control scheme in normal operation conditions, the following test scenario with several test cases are numerically investigated:

- Test Scenario 3: voltage regulation in operation conditions, where there is no fault, the DER is gridconnected functional, and different types and values of load changes in different buses and phases happen, as presented and analyzed through the following test cases. 
4) A sudden change of (1 MW, 0.32 Mvar) in the three-phase load at Bus 844 is observed.

5) An unbalanced load of $0.62 \mathrm{MW}$ is added to Bus 824, in both Phase A and Phase B.

6) An unbalanced load of (0.06 MW, 0.04 Mvar) is simultaneously added to both Phase B and Phase C at Bus 830.

7) An unbalanced load of (0.1 MW, 0.04 Mvar) is simultaneously added to both Phase B and Phase C at Bus 856.

8) A three-phase balanced load of (0.06 MW, $0.01 \mathrm{MW}$ ) is added to Bus 860 .

All the studied test cases elaborated above in each test scenario are listed in Table 2.

\subsection{Numerical analysis and discussions}

In Case 1, the grid protection devices successfully isolate the fault. The proposed voltage control scheme is applied at the DER-hosting Bus 852 and its promising performance globally on the voltage profile across the grid is particularly verified at the measurement Bus 850, as demonstrated in Fig. 11 where $V_{\mathrm{rms}, \mathrm{A}}, V_{\mathrm{rms}, \mathrm{B}}, V_{\mathrm{rms}, \mathrm{C}}$ are root mean square (RMS) values of voltage at phase $\mathrm{A}, \mathrm{B}$ and C. Following a single-line-to-ground (SLG) fault at Bus 820, the corresponding load in phase A drops at $t=0.5 \mathrm{~s}$, as the normally-closed switch $\mathrm{S} 1$ is opened to isolate the branch from Bus 818 to Bus 822. An overvoltage appears at Phase $\mathrm{A}$ in the voltage profile at the measurement Bus 850 in Fig. 11a, and therefore, the DER decreases its output power in Fig. 11c, d accordingly. In this case, however, the overvoltage at Bus 850 , while being mitigated a bit via the proposed control scheme, is still noticeable $(>0.5 \mathrm{kV})$ in Fig. 11a, b. The power through the DC-link decreases and this can be seen in Fig. 12. The faulted section of the grid is re-connected online at $t=0.9 \mathrm{~s}$, and one can see in Fig. 11 that the grid has

Table 2 Test scenarios and test case settings

\begin{tabular}{llllll}
\hline Test scenario & Case & Location & Phase & Type & Load \\
\hline Fault & 1 & Bus 820 & A & LG & - \\
& 2 & Line 834-858 & ABC & $3 \Phi G$ & - \\
& 3 & Bus 890 & ABC & $3 \Phi G$ & - \\
Load change & 4 & Bus 844 & ABC & - & $-1-\mathrm{j} 0.32$ \\
& 5 & Bus 824 & AB & - & 0.62 \\
& 6 & Bus 830 & BC & - & $0.12+\mathrm{j} 0.04$ \\
& 7 & Bus 856 & AC & - & $0.2+\mathrm{j} 0.08$ \\
& 8 & Bus 860 & ABC & - & $0.06+\mathrm{j} 0.02$
\end{tabular}

Note: negative value reflects a decrease in load; $\mathrm{G}$ stands for groundconnected

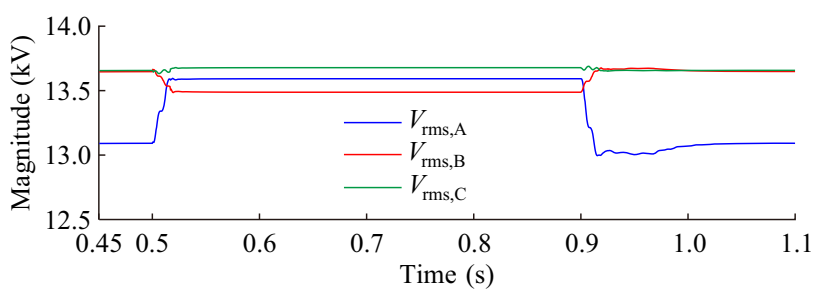

(a) At Bus 850 without proposed control scheme

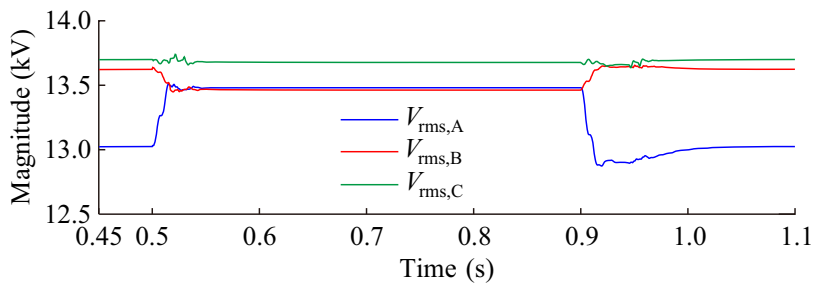

(b) At Bus 850 with proposed control scheme

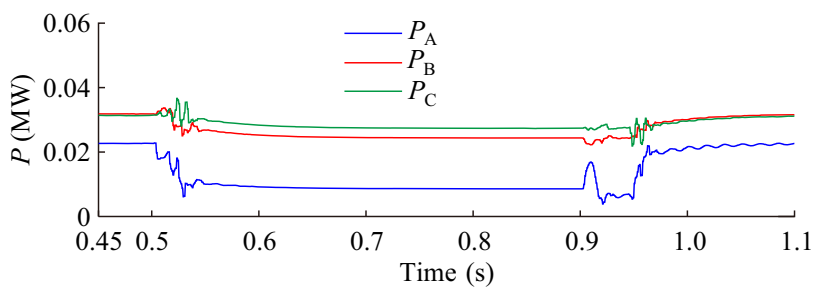

(c) At Bus 852 for active power

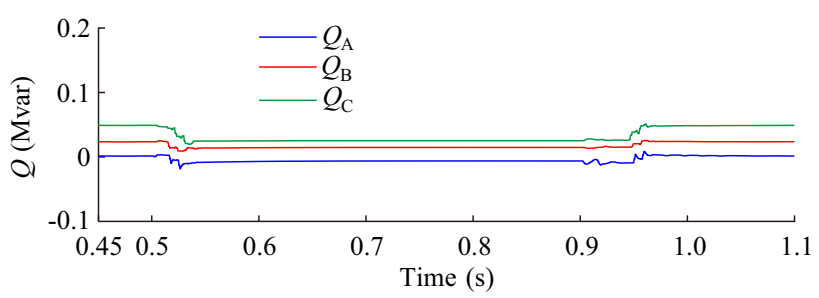

(d) At Bus 852 for reactive power

Fig. 11 Case 1 results

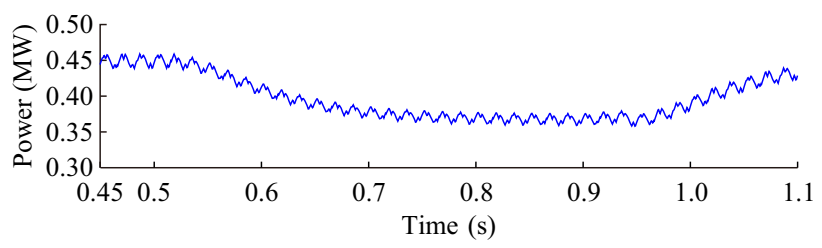

Fig. 12 Power supported by DC-link within the PE-interfaced DER in Case 1

returned back to its normal operation condition in Fig. 13.

In Case 2, a three-phase fault is simulated which triggers the normally-closed switch S2 to be opened and isolate the faulted section of the grid. In this case, a sudden load loss occurs at $t=0.5 \mathrm{~s}$ resulting in an overvoltage condition at Bus 850 in Fig. 14a, the proposed control scheme is applied, and the DER initially decreases its output power, as demonstrated in Fig. 14c, d. After 0.08 s, the DER starts absorbing power from the grid, as a result of which, the voltage starts to drop in Fig. 14b. Comparing the result 


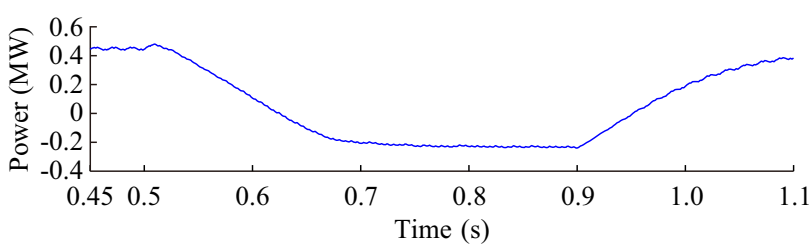

Fig. 13 Power supported by DC-link within the PE-interfaced DER in Case 2

observed in Case 2 with that one in Case 1 in Fig. 11b, we can see that in the former, some voltage distortions appear after the DER control is applied, reflecting the fact that the DER reaches its full potential to deal with the exceeded voltage in this case; according to Fig. 13, the DC-link starts draining power from the grid after $0.62 \mathrm{~s}$ of the fault occurrence.

In Case 3, a high-impedance fault occurs at a lowvoltage Bus 890 which is close to the DER location at Bus 852. The performance of the proposed DER voltage control

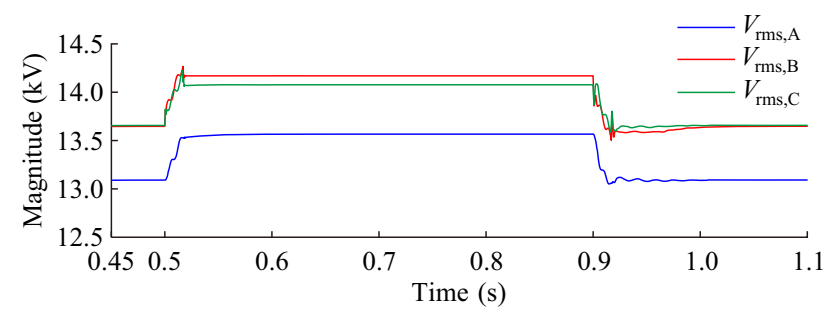

(a) At Bus 850 without proposed control scheme

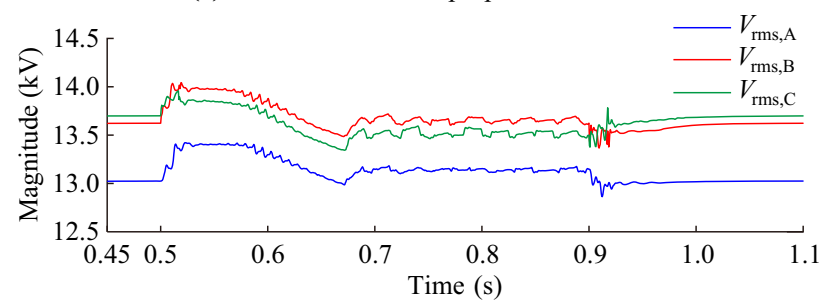

(b) At Bus 850 with proposed control scheme

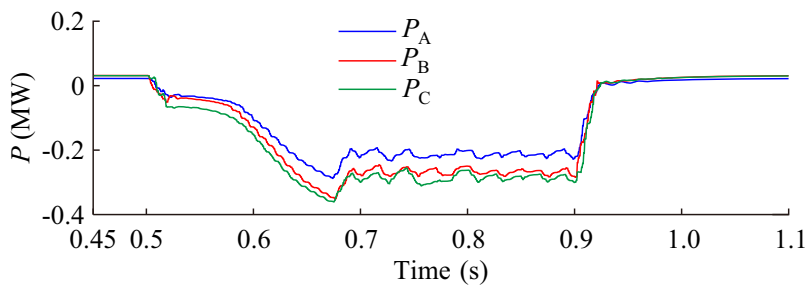

(c) At Bus 852 for active power

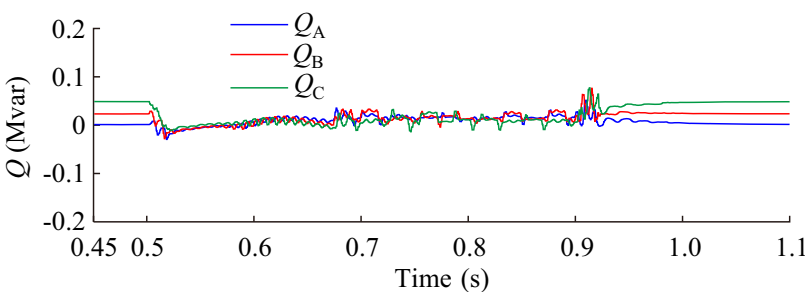

(d) At Bus 852 for reactive power scheme is verified as demonstrated in Fig. 15: following the fault, a voltage drop of around $1 \mathrm{kV}$ is observed in each phase at both DER location (Bus 852) in Fig. 15b and the measurement Bus 850 in Fig. 15a; the proposed DER control scheme was activated to regulate the voltage levels accordingly, the performance of which is demonstrated in Fig. 15c, d for Bus 850 and Bus 852, respectively. Comparing the results presented in $15 \mathrm{c}, \mathrm{d}$, where the proposed DER voltage control scheme is applied, we can see that the local voltage at Bus 852 where the DER is connected to is attributed a much better voltage regulation outcome than that of the measurement point at Bus 850. This observation verifies how effective the proposed MPC-based DER control performs ensuring the local voltage resilience in the distribution system.

Under the grid normal operation conditions with load variations in Case 4, the voltage control outcome is demonstrated in Fig. 16. With a significant load change observed at Bus 844, the voltage will subsequently increase

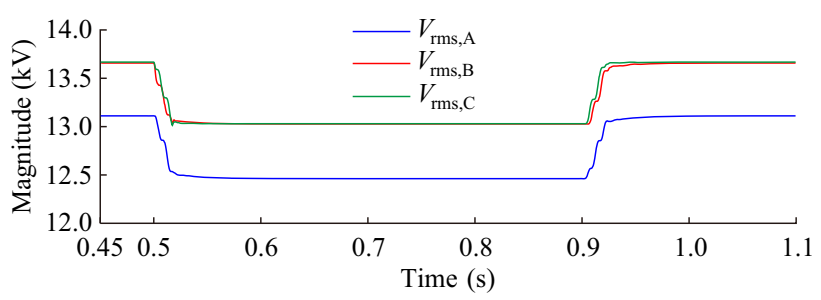

(a) At Bus 850 without proposed control scheme

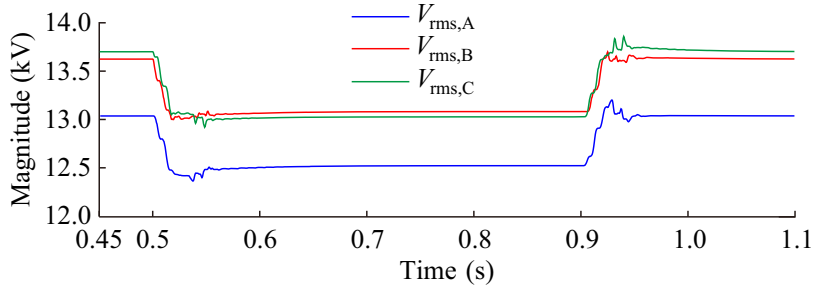

(b) At Bus 850 with proposed control scheme

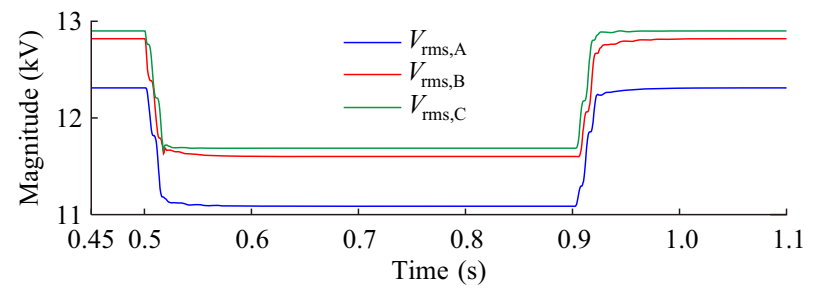

(c) At Bus 852 without proposed control scheme

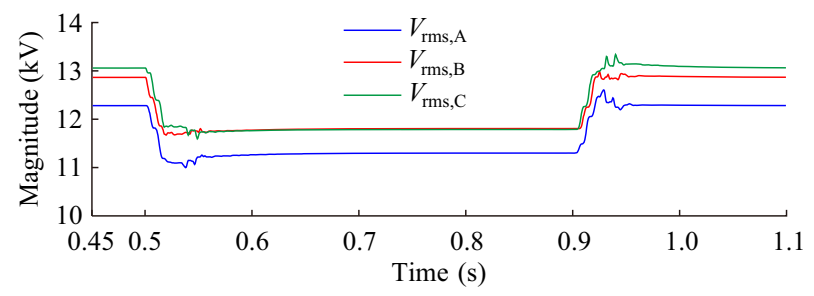

(d) At Bus 852 with proposed control scheme

Fig. 15 Case 3 results

Fig. 14 Case 2 results 


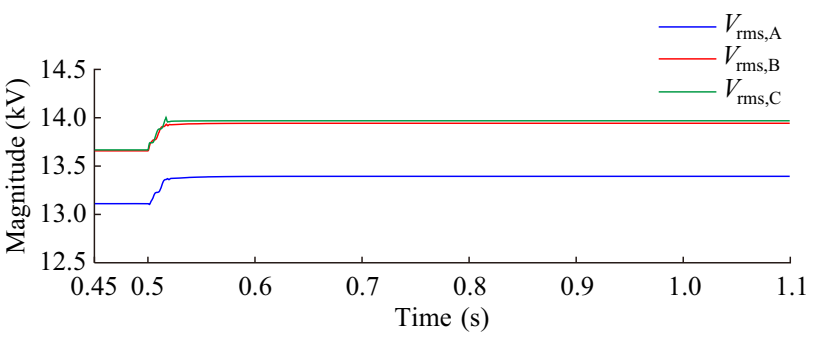

(a) At Bus 850 without proposed control scheme

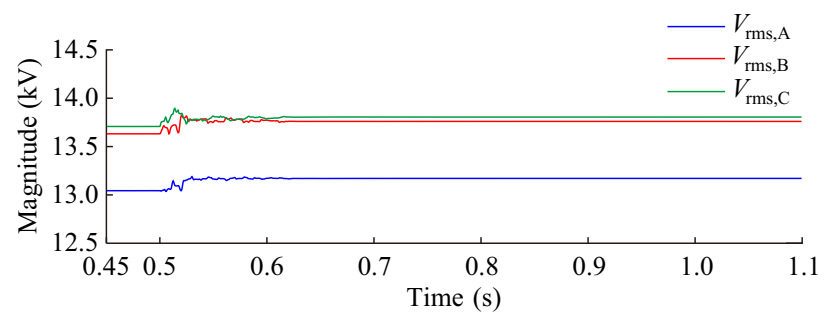

(b) At Bus 850 with proposed control scheme

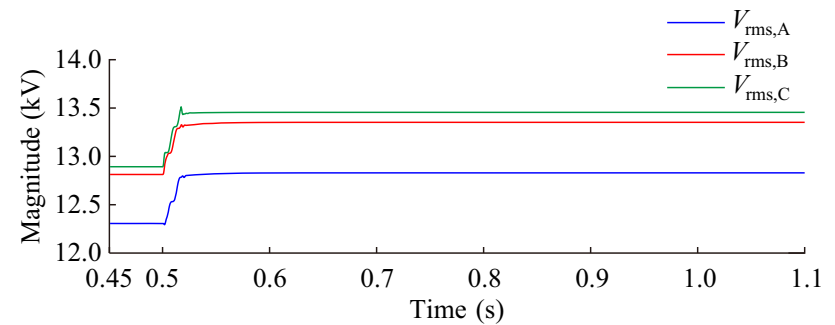

(c) At Bus 852 without proposed control scheme

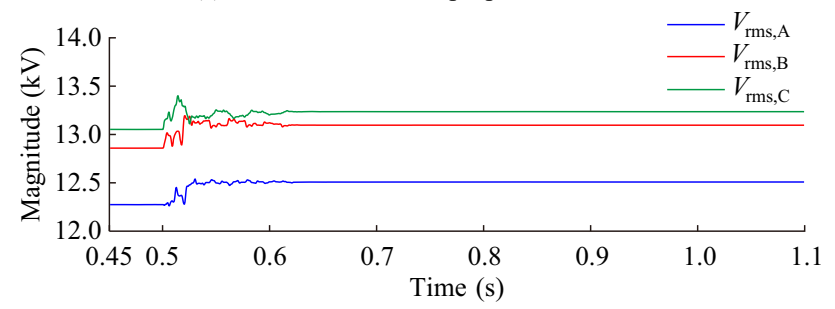

(d) At Bus 852 with proposed control scheme

Fig. 16 Case 4 results

as demonstrated in Fig. 16a, c at Bus 850 and Bus 852, respectively. With the proposed control scheme applied, Fig. $16 \mathrm{~b}$ demonstrates a promising performance at the measurement point (Bus 850) which is observed to be similar to that of the DER Bus 852. From the observations made in Case 4, we can conclude how effective the proposed control scheme can regulate the voltage locally and globally across the grid. In order to highlight the dynamic response speed of the proposed control scheme, a threephase PWM inverter with a classic proportional-integralderivative (PID) controller is applied and its performance is compared with that of the proposed solution. The PID control is tuned to have a fast response for the sake of fairness. Comparing the results in Fig. 16b, d with those presented in Fig. 17a, b, we can see that the proposed control scheme stands out at the response speed with the cost of higher distortions, while the PID control mechanism

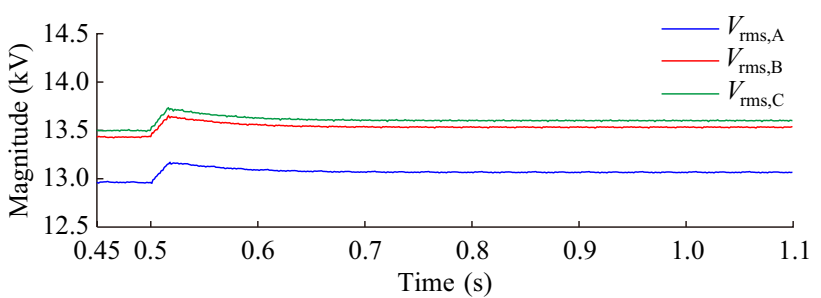

(a) At Bus 850

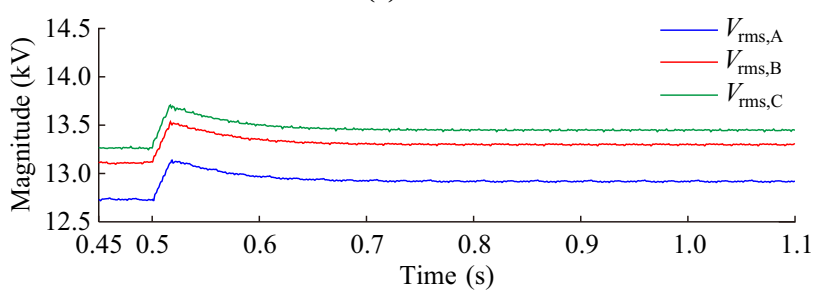

(b) At Bus 852

Fig. 17 Case 4 classical PID control results

brings in a smoother transient but is still slower even it is tuned to be faster than its traditional settings. Obviously, the benefits realized by deployment of the proposed scheme is highlighted through a significant reduction in the time interval that the grid connected devices are exposed to over voltage conditions during emergencies, which is critical when resilience to such violations is the focus of concern.

Similar analyses have been conducted in the other Cases 5-8 (introduced earlier in Section 5.2) and the performance of the suggested MPC-based DER control scheme in all cases is summarized in Table 3. We can see that in Cases where a load variation appears, no matter how it is balanced or unbalanced and major or minor, the proposed control scheme could resiliently regulate the voltages across the network. The degradation observed in the voltage profile is mitigated during abnormal condition, which improves the voltage resilience performance according to the evaluation framework described in Fig. 1. In all, it is demonstrated that the proposed MPC-based mechanism not

Table 3 Simulation results of voltage variations in different cases

\begin{tabular}{lrrrrrrrr}
\hline Case & \multicolumn{2}{c}{ Without proposed scheme } & & \multicolumn{3}{l}{ With proposed scheme } \\
\cline { 2 - 3 } \cline { 7 - 8 } & Phase A & Phase B & Phase C & & Phase A & Phase B & Phase C \\
\hline 1 & 0.034 & -0.013 & 0.001 & & 0.031 & -0.012 & -0.001 \\
2 & 0.032 & 0.036 & 0.029 & & 0.005 & 0.002 & -0.014 \\
3 & -0.045 & -0.043 & -0.044 & -0.035 & -0.038 & -0.047 \\
4 & 0.019 & 0.020 & 0.021 & & 0.009 & 0.009 & 0.007 \\
5 & -0.020 & -0.040 & -0.001 & -0.013 & -0.040 & -0.002 \\
6 & 0.002 & -0.010 & -0.007 & & 0.009 & -0.011 & -0.008 \\
7 & -0.013 & 0.006 & -0.018 & -0.006 & 0.006 & -0.021 \\
8 & -0.008 & -0.008 & -0.008 & -0.002 & -0.008 & -0.008 \\
\hline
\end{tabular}

Note: a negative value represents a voltage decrease 
only achieves the prescribed goals of resilience (i.e., supplying power and preventing damage to DERs from external faults), but also reduces the negative impacts on the adjacent equipment. Consequently, the power-controlled DER could promisingly support the grid performance locally.

In order to ensure the functionality of the designed predictor, the dynamic modeling of the inverter and associated LC filter (PE interface) is critical. In the cases of extreme events particularly when facing the inverter failure or LC filter failure, the performance of the predictor may be compromised due to the variations in critical parameters of the PE-interface. Hence, a robust and adaptive coordination of the predictor and the optimizer is needed to ensure a local and global resilience during extreme emergencies. Note that as long as the grid does not collapse, and the PE-interfaced DERs are neither damaged nor tripped from the grid during extreme events (i.e., can access the grid measurements), the proposed MPC predictor can still support the system and improve the voltage resilience.

\section{Conclusion}

This paper proposes a MPC-based strategy for PE-interfaced DERs in power distribution systems. The suggested control mechanism ensures a continuously-regulated voltage profile in normal operation conditions in the grid with load variation scenarios as well as voltage resilience in disturbances and abnormal operation conditions. Simulations on the IEEE 34-bus test feeder with multiple cases revealed that the proposed scheme is able to secure an acceptable performance of the voltage source inverter in faults and unbalanced conditions, potentially immunizing the DC source from damages even without a proper operation of the network protective devices. A sufficient number of DERs deployed in the network equipped with the proposed control mechanism could help realize a significantly-improved voltage recovery and resilience locally and globally.

Future work needs be focused on improving the robustness of this control scheme as the PE-interface dynamic models vary over time and the PE-interface itself is subject to failure. The control scheme must be able to tolerate the system aging and dynamic variations over time. Additionally, the functionality of acting as the main power source equipped with frequency and voltage control during islanding operation can be added so as to achieve an even more flexible control strategy.
Open Access This article is distributed under the terms of the Creative Commons Attribution 4.0 International License (http:// creativecommons.org/licenses/by/4.0/), which permits unrestricted use, distribution, and reproduction in any medium, provided you give appropriate credit to the original author(s) and the source, provide a link to the Creative Commons license, and indicate if changes were made.

\section{References}

[1] National Academies of Sciences, Engineering, and Medicine (2017) Enhancing the resilience of the nation's electricity system. National Academies Press, Washington

[2] Panteli M, Trakas DN, Mancarella P et al (2017) Power systems resilience assessment: hardening and smart operational enhancement strategies. Proc IEEE 105(7):1202-1213

[3] Farzin H, Fotuhi-Firuzabad M, Moeini-Aghtaie M (2016) Enhancing power system resilience through hierarchical outage management in multi-microgrids. IEEE Trans Smart Grid 7(6):2869-2879

[4] Wang X, Pournaras E, Kooij RE et al (2014) Improving robustness of complex networks via the effective graph resistance. Eur Phys J B 87(9):1-12

[5] Barus D (2011) Implementation of free governor action in power plant to increase system resilience of Jawa Bali power system network. In: Proceedings of international universities' power engineering conference, Soest, Germany, 5-8 September 2011, 5 pp

[6] Liu Y, Wu Q, Zhou X (2016) Co-ordinated multiloop switching control of DFIG for resilience enhancement of wind power penetrated power systems. IEEE Trans Sustain Energy 7(3):1089-1099

[7] Eshghi K, Johnson BK, Rieger CG (2015) Power system protection and resilient metrics. In: Proceedings of resilience week, Philadelphia, USA, 18-20 August 2015, 8 pp

[8] Ly T, Moura J, Velummylum G (2015) Assessing the bulk power system's resource resilience to future extreme winter weather events. In: Proceedings of IEEE PES general meeting, Denver, USA, 26-30 July 2015, 4 pp

[9] Krishnamurthy V, Kwasinski A (2016) Effects of power electronics, energy storage, power distribution architecture, and lifeline dependencies on microgrid resiliency during extreme events. IEEE J Emerg Sel Top Power Electron 4(4):1310-1323

[10] Allam MA, Hamad AA, Kazerani M et al (2018) A novel dynamic power routing scheme to maximize loadability of islanded hybrid AC/DC microgrids under unbalanced AC loading. IEEE Trans Smart Grid 9(6):5798-5809

[11] Wang P, Lu X, Yang X et al (2016) An improved distributed secondary control method for DC microgrids with enhanced dynamic current sharing performance. IEEE Trans Power Electron 31(9):6658-6673

[12] Meng L, Shafiee Q, Trecate GF et al (2017) Review on control of DC microgrids and multiple microgrid clusters. IEEE J Emerg Sel Top Power Electron 5(3):928-948

[13] De Brabandere K, Bolsens B, Van den Keybus J et al (2007) A voltage and frequency droop control method for parallel inverters. IEEE Tran Power Electron 22(4):1107-1115

[14] Guerrero JM, Hang L, Uceda J (2008) Control of distributed uninterruptible power supply systems. IEEE Trans Ind Electron 55(8):2845-2859 
[15] Wu TF, Chen YK, Huang YH (2000) 3C strategy for inverters in parallel operation achieving an equal current distribution. IEEE Trans Ind Electron 47(2):273-281

[16] Nasirian V, Shafiee Q, Guerrero JM et al (2016) Droop-free distributed control for AC microgrids. IEEE Trans Power Electron 31(2):1600-1617

[17] Lee CT, Chu CC, Cheng PT (2013) A new droop control method for the autonomous operation of distributed energy resource interface converters. IEEE Trans Power Electron 28(4):1980-1993

[18] Siri K, Lee C, Wu TE (1992) Current distribution control for parallel connected converters. IEEE Trans Aerosp Electron Syst 28(3):829-840

[19] NERC (2015) 2015 risk element: extreme physical events. http://www.nerc.com/pa/comp/Documents/2015\%20Extreme\% 20Physical\%20Events\%20v1.08.pdf. Accessed 21 January 2017

[20] Dehghanian P, Zhang B, Dokic T et al (2019) Predictive risk analytics for weather-resilient operation of electric power systems. IEEE Trans Sustain Energy 10(1):3-15

[21] Zhang B, Dehghanian P, Kezunovic M (2019) Optimal allocation of PV generation and battery storage for enhanced resilience. IEEE Trans Smart Grid 10(1):535-545

[22] Dehghanian P (2017) Power system topology control for enhanced resilience of smart electricity grids. Ph.D. thesis, Texas A\&M University

[23] Gholami A, Aminifar F, Shahidehpour M (2016) Front lines against the darkness: enhancing the resilience of the electricity grid through microgrid facilities. IEEE Electrif Mag 4(1):18-24

[24] Berkeley A, Wallace M, COO C (2010) Final report and recommendations by the council. National Infrastructure Advisory Council

[25] Haimes YY (2009) On the definition of resilience in systems. Risk Anal: Int J 29(4):498-501

[26] White House (2013) Critical infrastructure security and resilience. White House, Washington

[27] Cutter SL, Ahearn JA, Amadei B et al (2013) Disaster resilience: a national imperative. Environ: Sci Policy Sustain Dev 55(2):25-29

[28] Perrings C (2006) Resilience and sustainable development. Environ Dev Econ 11(4):417-427

[29] Hollnagel E, Woods DD, Leveson N (2007) Resilience engineering: concepts and precepts. Ashgate Publishing Ltd, Farnham

[30] Adger WN (2000) Social and ecological resilience: are they related? Prog Hum Geogr 24(3):347-364

[31] Jen E (2003) Stable or robust? What's the difference? Complexity $8(3): 12-18$

[32] Arghandeh R, von Meier A, Mehrmanesh L et al (2016) On the definition of cyber-physical resilience in power systems. Renew Sustain Energy Rev 58:1060-1069

[33] Lee JH (2011) Model predictive control: review of the three decades of development. Int J Control Autom Syst 9(3):415-424

[34] Morari M, Lee JH (1999) Model predictive control: past, present and future. Comput Chem Eng 23(4-5):667-682

[35] Rodriguez J, Kazmierkowski MP, Espinoza JR et al (2013) State of the art of finite control set model predictive control in power electronics. IEEE Trans Ind Inform 9(2):1003-1016

[36] Cortés P, Kazmierkowski MP, Kennel R et al (2008) Predictive control in power electronics and drives. IEEE Trans Ind Electron 55(12):4312-4324

[37] Kouro S, Cortés P, Vargas R et al (2009) Model predictive control a simple and powerful method to control power converters. IEEE Trans Ind Electron 56(6):1826-1838
[38] Huisman H, Roes M, Lomonova E (2016) Continuous control set space vector modulation for the $3 \times 3$ direct matrix converter. In: Proceedings of European conference on power electronics and applications, Karlsruhe, Germany, 5-9 September 2016, $10 \mathrm{pp}$

[39] Vazquez S, Rodriguez J, Rivera M et al (2017) Model predictive control for power converters and drives: advances and trends. IEEE Trans Ind Electron 64(2):935-947

[40] Lim CS, Levi E, Jones M et al (2014) FCS-MPC-based current control of a five-phase induction motor and its comparison with PI-PWM control. IEEE Trans Ind Electron 61(1):149-163

[41] Pigazo A, Moreno VM, Estbanez EJ (2009) A recursive park transformation to improve the performance of synchronous reference frame controllers in shunt active power filters. IEEE Trans Power Electron 24(9):2065-2075

[42] Duesterhoeft WC, Schulz MW, Clarke E (1951) Determination of instantaneous currents and voltages by means of alpha, beta, and zero components. Trans Am Inst Electr Eng 70(2):1248-1255

[43] Feola L, Langella R, Testa A (2013) On the effects of unbalances, harmonics and interharmonics on PLL systems. IEEE Trans Instrum Meas 62(9):2399-2409

[44] Chung SK (2000) A phase tracking system for three phase utility interface inverters. IEEE Trans Power Electron 15(3):431-438

[45] Zhang R, Prasad VH, Boroyevich D, Lee FC (2002) Threedimensional space vector modulation for four-leg voltagesource converters. IEEE Trans Power Electron 17(3):314-326

Shiyuan WANG received the B.E. degree in mechanical engineering from University of Science and Technology Beijing, China, in 2012; the M.Sc. degree in electrical engineering from The George Washington University, Washington DC, USA, in 2014. He is currently pursuing the Ph.D. degree at the Department of Electrical and Computer Engineering, George Washington University,Washington DC, USA. His research interests include power system reliability and resiliency, smart grid and renewable energy, power grid harmonic analysis, and application of signal processing in energy analytics.

Payman DEHGHANIAN received the B.Sc., M.Sc., and Ph.D. degrees all in electrical engineering from the University of Tehran, Tehran, in 2009, Sharif University of Technology, Tehran, in 2011, and Texas A\&M University, College Station, USA, in 2017, respectively. $\mathrm{He}$ is currently an Assistant Professor with the Department of Electrical and Computer Engineering, George Washington University, Washington DC, USA. His research interests include power system protection and control, power system reliability and resiliency, asset management, and smart electricity grid applications. Dr. Dehghanian is the recipient of the 2013 IEEE Iran Section Best M.Sc. Thesis Award in Electrical Engineering, the 2014 and 2015 IEEE Region 5 Outstanding Professional Achievement Awards, and the 2015 IEEE-HKN Outstanding Young Professional Award.

Mohannad ALHAZMI received the B.Sc. and M.Sc. degrees in electrical engineering from Umm Al-Qura University, Saudi Arabia in 2013 and The George Washington University, Washington DC, USA, in 2017, respectively. He is currently pursuing his Ph.D. degree in the Department of Electrical and Computer Engineering at The George Washington University. His research interests include power 
system control, reliability and resiliency, cyber security and smart electricity grid applications.

Mostafa NAZEMI received the B.Sc. degree from K. N. Toosi University of Technology, Tehran, Iran, in 2015, and the M.Sc. degree from Sharif University of Technology, Tehran, Iran, in 2017, in electrical engineering and in energy systems engineering, respectively. $\mathrm{He}$ is currently pursuing the Ph.D. degree in electrical engineering at the Department of Electrical and Computer Engineering, George Washington University, Washington DC, USA. His research interests include power system resilience, power system planning and operation, energy optimization, and smart electricity grid applications. 